\title{
Impact of Consumers' Self-Construal, Empathy, and Moral Identity on Purchase Intention toward Cause-Related Marketing Goods: A Perspective of the Moderated Mediation Model
}

\author{
Hsin-Ti Yang1, Ghi-Feng Yen ${ }^{2 *}$ \\ ${ }^{1}$ Department of Business Administration, Chien Hsin University of Science and Technology, Taoyuan City, Taiwan \\ ${ }^{2}$ Department of Business Administration, Chung Yuan Christian University, Taoyuan City, Taiwan \\ Email: cindyyang@uch.edu.tw, *gfyen@cycu.edu.tw
}

How to cite this paper: Yang, H.-T., \& Yen, G.-F. (2018). Impact of Consumers' Self-Construal, Empathy, and Moral Identity on Purchase Intention toward Cause-Related Marketing Goods: A Perspective of the Moderated Mediation Model. Psychology, 9, 260279.

https://doi.org/10.4236/psych.2018.92017

Received: January 23, 2018

Accepted: February 25, 2018

Published: February 28, 2018

Copyright $\odot 2018$ by authors and Scientific Research Publishing Inc. This work is licensed under the Creative Commons Attribution International License (CC BY 4.0).

http://creativecommons.org/licenses/by/4.0/

\section{Abstract}

This study examined whether the impact of consumers' self-construal on purchase intention toward cause-related marketing (CRM) goods was indirectly affected by empathy and moral identity. A total of 524 valid samples were collected, after which the moderated mediation hypotheses were tested using Process software. The research data were segmented into three score groups to compare the differences between the high score and low score groups. The empirical results revealed the following findings. 1) Moral identity internalization and symbolization resulted in positive moderation effects on the direct impact of interdependent self-construal on purchase intention toward CRM products. However, under the mediating effect of empathy, the "low/low" and "high/high" groups from moral identity internalization and symbolization each produced positive and negative moderation effects. 2) Regarding the direct impact of moral identity internalization and symbolization on independent self-construal toward purchase intention toward CRM products, the "low/low" and "low/high" groups from internalization and symbolization produced positive moderation effects. However, under the mediating effect of empathy, only the "low/low" groups from internalization and symbolization produced significant positive mediating effects. Within the consumers' self-construal and its impact on purchase intention toward CRM products, the mediation role of empathy and moderated mediation role of the two moral identity dimensions in the influence relationship were constructed. In terms of practical aspects, companies are recommended to conduct market segmentation based on consumers' moral identities and develop distinct 
marketing strategies accordingly. Future studies are recommended to explore the factors behind the impact of these two groups from moral identity on purchase intention toward CRM products.

\section{Keywords}

Consumers' Self-Construal, Purchase Intention, Empathy, Moral Identity, Cause-Related Marketing, Moderated Mediation Model, Psychological Aspects

\section{Introduction}

In recent years, companies have been implementing corporate social responsibility (CSR) as a strategic tool for achieving financial goals and enhancing reputation (Skarmeas \& Leonidou, 2013). Promoting moral standards through CSR to improve corporate image strengthens consumers' attitudes toward the brand and products of a company. Companies can also have a social impact by supporting charitable donations or through cause-related marketing (CRM), which can improve their visibility and prompt them to invest substantial effort in charitable causes to communicate their efforts. Past researches have empirically established that CSR initiatives enhance consumer charitable behaviors and in turn to lead to positive outcomes (Sen and Bhattacharya, 2001). However, more precisely understanding of the underlying processes driving consumers' responses is still needed.

Regarding cognitive perspectives, the self-system is dynamic and individuals' self-construal-related decisions are made based on their other contemporary social identities and relevant factors (Oyserman, 2009). However, the findings of related previous studies diverge on whether consumers with independent selfconstrual and interdependent self-construal prefer to make purchase decisions based on emotional or cognitive aspects (Hong \& Lee, 2010; Hong \& Chang, 2015). Therefore, other relevant factors related to the impact of self-construal on consumer decision-making should be explored. According to Kim and Johnson (2013), moral emotions affect purchase intentions toward CRM products and influence charitable acts (Arnett et al., 2003; Hoffman, 1981). Because empathy is a moral emotion (Haidt, 2003), it promotes altruistic behavior (Batson, 1987; Eisenberg \& Miller, 1987) and mediates the relationship between moral identity and charitable contributions (Lee et al., 2014). As Taiwanese society is based on the ethnic Chinese culture of collectivism, other-focused moral emotions have a significant impact on purchase intention toward CRM products.

Because CRM is a unique cause linking product sales to support of charities (Varadarajan \& Menon, 1998), consumers purchasing cause-related products represents consumers' support for charities and is regarded as an act of moral sensitivity and a behavior that demonstrates consumer identities (Barone \& Roy, 2010; Lafferty \& Goldsmith, 2005). Therefore, consumer purchases of cause-related goods denote that the consumers regard themselves as individuals with moral 
considerations, because CRM prompts consumers to demonstrate their social identity (Reed, 2004), of which moral identity is one aspect (He et al., 2016). Previous studies have verified that moral identity is influenced by cognitive and emotional factors (He et al., 2016). The implementation of CSR facilitates consumers in experiencing moral emotions and produces behavioral responses (e.g., negative responses such as negative word-of-mouth, complaints, and boycotts; or positive responses such as positive word-of-mouth, identification, and investment) (Xie et al., 2015). However, few studies have explored the impact of moral identity on "indirect" donations (e.g., purchasing CRM products) (He et al., 2016).

Using a social cognitive model, Aquino, Freeman, Reed, Lim, and Felps (2009) proposed that situational factors and moral identities present interactive effects on moral behaviors. In other words, situational factors can drive moral behaviors by enhancing moral identity within the self-concept. Numerous studies have noted that under the influence of the collectivist culture, the ethnic Chinese are situation oriented, which means that they are more capable of displaying distinct attitudes and behaviors in different social contexts than are westerners, thereby demonstrating an interdependent self-construal ( $\mathrm{Lu}, 2003)$. He et al. (2016) verified that moral identity internalization affects consumers' reaction behaviors toward CRM and is affected by cognitive and emotional variables. Seeing others' misfortune triggers an individual's empathic response to care for others (Tangney et al., 2007) because emotional empathy is regarded as the center of humans' moral emotional system (Eisenberg et al., 2004). As CRM is usually linked to social or ethical causes (Ross et al., 1992; Varadarajan \& Menon, 1988), based on the research recommendations of $\mathrm{He}$ et al. (2016), the present study included empathy as one of the moral emotional variables in the discussion and proposed self-construal as an antecedent variable affecting moral emotion. In addition, on the basis of the research design of Winterich, Mittal, and Aquino (2013), this study explored whether consumers' self-construal under the collectivist culture combined with empathy and moral identity influenced purchase intention toward CRM products.

\section{Literature Review}

\subsection{CRM and Self-Construal}

Previous studies have proposed that CRM consists of two goals: promoting corporate performance and assisting charities. CRM has been defined as "the linking of charity fundraising through purchasing corporate goods or services," or in other words, corporate contributions to charities originate from CRM product sales rather than the operational budgets of corporations (Varadarajan \& $\mathrm{Me}$ non, 1988). Studies have verified that CRM improves product sales (Andrew et al., 2014), strengthens consumer attitudes toward companies that sponsor causes (Ross et al., 1992), increases product sales prices (Leszczyc \& Rothkopf, 2010), and enhances the positive image of other products under the same brand (Krishna 
\& Rajan, 2009). CRM also promotes relationships between stakeholders (Bhattacharya et al., 2009) and enhances a firm's positive word of mouth (Thomas et al., 2011). However, CRM also has negative effects; for example, skepticism and distrust can cause consumers to develop a perception of "cause-exploitative marketing" toward poorly designed charitable strategies implemented by companies (Sasse \& Trahan, 2007; Varadarajan \& Menon, 1988). Forehand and Grier (2003) verified that consumers dislike companies' attempts to demonstrate pure public service motivations while concealing their strategic earnings behind charities. Likewise, studies have shown that consumers are more sensitive to real business charities (Lee et al., 2009) and feel averse to the ingratiation of companies (Godfrey, 2005).

Scholars have discovered that self-construal is the foundation of cognitive and emotional response systems, and people define their relationships with others according to different self-construals (Markus \& Kitayama, 1991; Shen, Wan, \& Wyer, 2011). Self-construal also dominates consumers' identification with charitable cause and affects their preferences toward promotional methods (Winterich \& Barone, 2011). Self-construal refers to how people view their relationships with others and the social environment, and from a psychological perspective is divided into "interdependent self-construal" and "independent self-construal" (Markus \& Kitayama, 1991). Such a contrasting concept of cultural dichotomy can be regarded as the correspondence of "individualism/collectivism" between the individual level and cultural level (Lu, 2003). Independent self-construal and interdependent self-construal are two sets of schemata that exist simultaneously (Markus \& Kitayama, 1991), with the former emphasizing individual and selfcentered analysis units and the latter emphasizing social and contextual selves as analysis units linked to society (Duclos \& Barasch, 2014).

Previous studies have verified that interdependent self-construal is positively correlated with charitable acts (Skarmeas \& Shabbir, 2011) and consumers with interdependent self-construal prefer CRM with discount promotions (Winterich \& Barone, 2011). Other studies have discovered that consumers with interdependent self-construal are more willing to pay higher prices for CRM products (Vaidyanathan et al., 2013). Hong and Chang (2015) believed that self-construal influences whether consumers make feeling-based or reason-based decisions; consumers with independent self-construal have higher preference for affectively superior options, whereas those with interdependent self-construal have higher preference for cognitively superior options. However, in another study, the results contradicted this, finding that individuals with independent self-construal prefer cognitively superior options and those with interdependent self-construal favor affectively superior options (Hong \& Lee, 2010). Because self-construal is shaped by cultural environments, a collectivist cultural background tends to produce individuals with interdependent self-construal, whereas an individualistic environment is prone to producing individuals with independent self-construal (Hofstede, 2001; Markus \& Kitayama, 1991; Singelis, 1994). Therefore, this study 
developed the following hypothesis:

H1: Consumers' self-construal affects their purchase intentions toward CRM products.

Hla: Independent self-construal affects purchase intention toward CRM products.

$H 1 b$ : Interdependent self-construal affects purchase intention toward CRM products.

\subsection{Self-Construal and Empathy}

In moral emotions, empathy refers to "emotions evoked by the suffering of others." Lazarus (1991) suggested that empathy not only constitutes a compassionate reaction that arises in response to others' misfortune but also involves sharing others' positive emotions. Thus, consumers' empathy may influence their responses to CRM. Emotional empathy is also regarded as the center of humans' "moral emotional system" (Eisenberg et al., 2004), because seeing others' misfortune triggers individuals' empathic response to care for others, and such empathy prompts individuals to assist the less fortunate people (Tangney et al., 2007). Therefore, empathy is a crucial prosocial capability that constitutes the ability to care for unfortunate individuals (Xie et al., 2015). Studies have also found that empathy encourages behaviors related to donation (Eisenberg \& Miller, 1987). Some scholars have proposed that empathy represents a donor's sympathy toward a particular recipient (Hoffman, 2000), and thus, differing charitable activities elicit distinct reaction behaviors because of consumers' empathy.

Previous studies have derived two emotional categories from independent and interdependent self-construal, namely ego-focused and other-focused emotions (Aaker \& Williams, 1998; Kitayama et al., 2006). Markus and Kitayama (1991) maintained that people from individualist backgrounds are more inclined to express ego-focused emotions such as pride and anger. Ego-focused emotions represent the inner states and attributes that are connected to the self, and thus exclude others while remaining consistent with the consciousness, experience, and expression of the self (Aaker \& Williams, 1998). By contrast, individuals from collectivist backgrounds tend to express other-focused emotions such as guilt, empathy, embarrassment, and shame (Aaker \& Williams, 1998; Kitayama et al., 2000, 2006; Markus \& Kitayama, 1991; Matsumoto, 1989), all of which are emotions that link individuals to close friends or others in society and help to reach a consensus on the need for unity, harmony, and connection with others (Aaker \& Williams, 1998). Because self-construal evokes distinct emotions based on different experiences of any given moment (Aaker \& Williams, 1998; Kitayama et al., 2006; Markus \& Kitayama, 1991; Matsumoto, 1989), moral emotions influence purchase intention toward CRM products (Kim \& Johnson, 2013). Additionally, as empathy promotes altruistic intentions (Batson, 1987; Eisenberg \& Miller, 1987), it also mediates the relationship between moral iden- 
tity and charitable contributions (Lee et al., 2014). This study asserted that the "causes" linked to CRM products evoke consumers' moral emotions and affect their purchase intentions. Thus, this study proposed the following hypotheses based on the literature review in this section:

H2: Consumers' self-construal affects their purchase intentions toward corporate CRM products through empathy.

H2a: Independent self-construal affects purchase intention toward corporate CRM products through empathy.

H2b: Interdependent self-construal affects purchase intention toward corporate CRM products through empathy.

\subsection{Two Dimensions of Moral Identity}

Aquino and Reed (2002) developed a definition of moral identity based on the social cognitive model and asserted that the network formed by associated moral traits, targets, and behaviors represents an individual's moral identity. In this associative network, the strength of moral connection reflects the degree of self-importance in an individual's moral identity. Moral identity is divided into two dimensions, namely the private dimension, known as internalization, and the public dimension, known as symbolization (Winterich, Mittal, \& Aquino, 2013). Studies have argued that individuals with high internalized moral identity are more susceptible to the influences of moral ideas on their moral traits, goals, and behaviors (Aquino \& Reed, 2002). In other words, individuals who believe that they have a high internalized moral identity are dedicated to moral actions, which include charitable acts (Aquino et al., 2009). The dimension of moral identity symbolization indicates individuals' intentions to convey their moral perspectives to the outside world through explicit behaviors that seek to gain identification and praise from others. In other words, individuals with high moral identity symbolization tend to convey personal moral goals or ideas so that others know about them, whereas those with low moral identity symbolization are less committed to such public display (Winterich, Mittal, \& Aquino, 2013). In particular, individuals with low internalized moral identity and high moral identity symbolization facilitate the emergence of prosocial behaviors upon receiving recognition from others (Winterich, Aquino, Mittal, \& Swartz, 2013).

In the interaction model of social cognitive theory, behavior, cognition, and other factors related to people and the environment exhibit a two-way influence (Bandura, 1989). Individuals' actions and changes are rooted in the social system, and thus their actions are deeply influenced by the wider social structure and their moral values are shaped over time in collectivist environments ( $\mathrm{Lu}$ et al., 2012). In Chinese society, moral identity internalization is nurtured and influenced by traditional Confucianism, which emphasizes ren and yi as the basic principles of life. In addition, individuals maintain self-consistency according to their own moral values and treat others in a reciprocal manner. Lu (2003) noted 
that the Chinese are situation oriented and more capable than westerners of demonstrating distinct values and attitudes based on different social contexts. The Chinese also pay special attention to "face work" which compels individuals to deliberately display certain behaviors to instill specific impressions in others in some contexts (Huang, 1987). Moral identity symbolization refers to an individual's attempt to convey the views of his or her personal moral identity to the outside world through behaviors (Winterich, Mittal, \& Aquino, 2013). Because individuals distinguish the relationships between themselves and others based on different types of self-construal (Markus \& Kitayama, 1991; Shen et al., 2011), the present study inferred that the two dimensions of moral identity strengthen the relationship between self-construal and purchase intention toward CRM products in Chinese collectivist contexts. Thus, the following hypotheses were proposed:

H3: Moral identity internalization strengthens the impact of consumers' selfconstrual on their purchase intentions toward CRM products.

H3a: Moral identity internalization strengthens the impact of independent self-construal on purchase intention toward CRM products.

H3b: Moral identity internalization strengthens the impact of interdependent self-construal on purchase intention toward CRM products.

H4: Moral identity symbolization strengthens the impact of consumers' selfconstrual on their purchase intentions toward CRM products.

H4a: Moral identity symbolization strengthens the impact of independent self-construal on purchase intention toward CRM products.

H4b: Moral identity symbolization strengthens the impact of interdependent self-construal on purchase intention toward CRM products.

Because the Chinese are influenced by traditional Confucianism, self-centered social behaviors must follow the established sociomoral order to emphasize the relational self (Ho, 1995). Studies have discovered that the Chinese emphasize "face work," which is a type of "impression management" in some situations (Huang, 1987) and is consistent with the traits of high moral identity symbolization (Winterich, Mittal, \& Aquino 2013). Other studies have investigated the direct impacts of moral identity internalization and symbolization on prosocial behaviors (Aquino \& Reed, 2002; Reed \& Aquino, 2003; Reed et al., 2007), as well as that of the interaction between moral identity internalization and symbolization on prosocial behaviors (Skarlicki et al., 2008). Winterich, Aquino, Mittal, and Swartz (2013) investigated a three-way interaction between moral identity internalization, moral identity symbolization, and recognition to explore the impact of this interaction on prosocial behaviors. Yen et al. (2017) discovered that moral identity symbolization mediates the relationship between a consumer's collectivist mindset and the intention to purchase fair trade products. In particular, this mediating effect significantly affects individuals with high moral identity symbolization.

The present study asserted that because situational factors strengthen moral 
behaviors (Aquino et al., 2009), in a collectivist environment, self-construal evokes other-focused emotions (i.e., empathy), together with the situational orientation common in Chinese culture ( $\mathrm{Lu}, 2003)$ and emphasis on the relational self (Ho, 1995). Therefore, moral identity internalization is expected to strengthen the mediating effect of empathy, thereby affecting purchase intention. In addition, since few studies have found symbolization significant ( $\mathrm{He}$ et al., 2016; Lee et al., 2014; Xie et al., 2015), and the "face work" emphasized by the Chinese represents explicit behaviors performed under specific circumstances to gain praise from others (Huang, 1987), moral identity symbolization is expected to strengthen the mediating effect of empathy. Accordingly, this study proposed the following hypotheses:

H5: The mediating effect of empathy on consumers' self-construal and purchase intention toward CRM products is strengthened by moral identity internalization.

H5a: The mediating effect of empathy on independent self-construal and purchase intention toward CRM products is strengthened by moral identity internalization.

H5b: The mediating effect of empathy on interdependent self-construal and purchase intention toward CRM products is strengthened by moral identity internalization.

H6: The mediating effect of empathy on consumers' self-construal and purchase intention toward CRM products is strengthened by moral identity symbolization.

H6a: The mediating effect of empathy on independent self-construal and purchase intention toward CRM products is strengthened by moral identity symbolization.

H6b: The mediating effect of empathy on interdependent self-construal and purchase intention toward CRM products is strengthened by moral identity symbolization.

The conceptual framework of this study consisting of all the aforementioned hypotheses is shown in Figure 1.

\section{Research Method}

\subsection{Research Participants and Data Collection Method}

In 2016, Taiwan, China was the highest ranked country for convenience store density, with every 2304 people having one convenience store to serve their needs (Common Wealth, 2016). Because CSR activities have become a part of the intense competition among convenience stores coupled with the common use of CRM for low-cost consumer goods (Chang \& Cheng, 2015), the present study adopted convenience store CRM as the main research subject.

A pretest was conducted on university students before the formal questionnaire survey was issued. The pretest questionnaire contained a description of a convenience store charity event that the respondents were required to read before answering the questions. After gaining an understanding of the nature of 


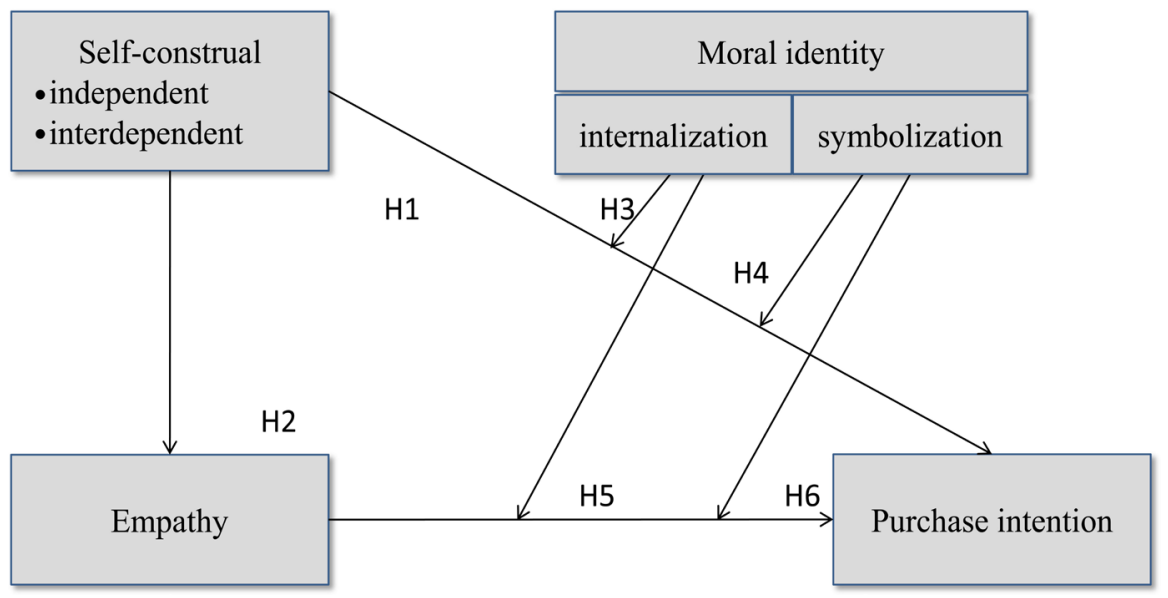

Figure 1. The conceptual framework.

the event, the students were requested to complete the questionnaire on purchase intention, which was measured using a 7-point Likert scale. Subsequently, the respondents were requested to answer questions on topics such as self-construal, moral identity, and empathy, and finally questions involving demographic data. Based on the pretest results, the questionnaire was revised and modified to ensure effective measurement. For administering the formal questionnaire, purposive sampling and convenience sampling was used. The formal questionnaire survey was conducted in the third quarter of 2016, during which communities and university faculties and students from four geographical regions (north, south, west, and east) in Taiwan were sampled, with 524 valid samples eventually received. The respondents' average age was 30 - 34 years. Most respondents were women (58.4\%) and listed their occupation as "other" (including the service industry and self-employed individuals (22.1\%)), followed by students $(19.8 \%)$ and traditional industries (19.8\%). The sampling data could be represented most consumers.

\subsection{Variable Measurement and Reliability Test}

For the research dimensions, this study adopted multiple measurement items from past studies. The self-construal dimension referenced the scale developed by Singelis (1994), which consists of 30 items that separately measure interdependent self-construal ( $\alpha=0.816)$ and independent self-construal $(\alpha=0.779)$. The empathy dimension was based on the designs of Eisenberg et al. (2004), Lazarus (1991), and Xie et al., (2015), and contained seven items $(\alpha=0.822)$. The moral identity dimension was derived from the 10-item self-importance of moral identity scale developed by Aquino and Reed (2002), wherein moral identity internalization $(\alpha=0.720)$ and symbolization $(\alpha=0.787)$ are each measured based on five items. The purchase intention dimension referenced the scale developed by White et al. (2012), which consists of four items. The questionnaire in the present study was measured using a 7-point Likert scale, with each item answered as one of seven options ranging from "strongly disagree" to "strongly 
agree" and assigned a score of $1-7(\alpha=0.931)$.

To confirm the reliability of the items in the questionnaire, this study conducted an initial pretest on 40 university students, after which items with an item-total correlation of lower than 0.3 were removed. Three items were removed, including two items from the independent self-construal dimension and one from the moral identity internalization dimension. The Cronbach's a values for the finalized dimensions were all greater than 0.7 with an overall reliability of 0.913 , indicating that the items in the questionnaire all possessed favorable reliability. In addition, this study referenced the study by Yen et al. (2017) to verify whether the two moral identity dimensions moderated the mediating effect of empathy. Following the recommendation of Kelly (1939), respondents with average scores lower than $27 \%$ in the two moral identity dimensions were assigned to the low score group, whereas those with average scores higher than $73 \%$ were assigned to the high score group. This splitting approach has been verified by numerous scholars such as Gelman and Park (2009), who verified that segmenting data into groups of $1 / 4-1 / 3$ achieves more satisfactory outcomes than segmenting data into groups of $1 / 2$. Similar research methods have also been verified as effective in studies by numerous psychometricians (Yen et al., 2017). Therefore, this study segmented the data into three score groups to compare the differences between the high score and low score groups.

Before testing our hypotheses, we ran confirmatory factor analysis (CFA) for our measures by using AMOS 18. Results of the CFA (Table 1) showed that the factor loadings of dimensions were all higher than 0.5 and statistically significant. All goodness of fit indicators for the measurement model were within acceptable ranges $\left(\chi^{2}=364.78 ; \mathrm{df}=155 ; \chi^{2} / \mathrm{df}=2.35 ; \mathrm{GFI}=0.93 ; \mathrm{AGFI}=0.91\right.$; NFI $=0.92$; CFI $=0.95$; RMSEA $=0.05)$. Therefore, the measurement model's convergent validity assessment meets the standards proposed by Anderson and Gerbing (1988).

\section{Research Results}

\subsection{Descriptive Statistics and Correlation Analysis}

Table 2 shows the means, standard deviations, and correlation coefficients for the variables in this study. Purchase intention toward CRM products was positively correlated with all the independent variables and achieved significance.

\subsection{Hypothesis Verification}

This study investigated whether consumers' purchase intention toward CRMproducts was affected by self-construal, moral identity, or empathy, with the moderated mediation hypotheses (Model 17, 5000 bootstrap resamples) proposed in this study tested using Process software (Hayes, 2013). The present study initially examined the direct effect of consumers' self-construal on purchase intention toward CRM products, after which the mediating effect of empathy 
Table 1. Results of confirmatory factor analysis.

\begin{tabular}{|c|c|c|c|c|}
\hline Items & Reliability ( $\alpha$ ) & Factor Loadings & CR & AVE \\
\hline Independence & 0.78 & & 0.64 & 0.37 \\
\hline I value being in good health above everything. & & 0.59 & & \\
\hline Being able to take care of myself is a primary concern for me. & & 0.52 & & \\
\hline My personal identity, independent of others, is very important to me. & & 0.71 & & \\
\hline Interdependence & 0.82 & & 0.76 & 0.53 \\
\hline $\begin{array}{l}\text { I often have the feeling that my relationships with others are more important than my own } \\
\text { accomplishments. }\end{array}$ & & 00.49 & & \\
\hline It is important to me to respect decisions made by the group. & & 00.80 & & \\
\hline It is important for me to maintain harmony with my group. & & 00.68 & & \\
\hline Moral Identity Internalization & 0.72 & & 0.76 & 0.53 \\
\hline It would make me feel good to be a person who has these characteristics. & & 0.83 & & \\
\hline Being someone who has these characteristics is an important part of who I am. & & 0.83 & & \\
\hline Having these characteristics is not really important to me. & & 0.50 & & \\
\hline Moral Identity Symbolization & 0.79 & & 0.74 & 0.50 \\
\hline $\begin{array}{l}\text { The types of things I do in my spare time (e.g., hobbies) clearly identify me as having these } \\
\text { characteristics. }\end{array}$ & & 0.86 & & \\
\hline The kinds of books and magazines that I read identify me as having these characteristics. & & 0.72 & & \\
\hline $\begin{array}{l}\text { The fact that I have these characteristics is communicated to others by my membership in } \\
\text { certain organizations. }\end{array}$ & & 0.50 & & \\
\hline Empathy & 0.82 & & 0.87 & 0.56 \\
\hline When I see someone being take advantage of, I feel kind of protective toward them. & & 0.70 & & \\
\hline When I see someone being treated unfairly, I sometimes feel pity for them. & & 0.84 & & \\
\hline I often have tender, concerned feelings for people less fortunate than me. & & 0.77 & & \\
\hline Sometimes I feel sorry for other people when they are having problems. & & 0.75 & & \\
\hline I am often quite touched by things that I see happen. & & 0.68 & & \\
\hline Purchase Intention & 0.93 & & 0.89 & 0.74 \\
\hline I would be likely to purchase this product. & & 0.86 & & \\
\hline I would likely make this product one of my first choices in this product category. & & 0.92 & & \\
\hline I would exert a great deal of effort to purchase this product. & & 0.78 & & \\
\hline
\end{tabular}

CR Composite Reliability, AVE Average Variance Extracted.

Table 2. Summary statistics and correlations

\begin{tabular}{|c|c|c|c|c|c|c|c|}
\hline Construct & (1) & (2) & (3) & (4) & (5) & Mean & SD \\
\hline 1) $P I$ & & & & & & 5.64 & 1.27 \\
\hline 2) IND & $0.291^{\star *}$ & & & & & 5.13 & 0.71 \\
\hline 3) INT & $0.316^{\star *}$ & $0.499^{* *}$ & & & & 5.17 & 0.68 \\
\hline 4) MII & $0.256^{\star *}$ & $0.347^{\star *}$ & $0.429^{* *}$ & & & 5.83 & 0.83 \\
\hline 5) MIS & $0.289^{* *}$ & $0.408^{\star *} 0$ & $0.474^{* *}$ & $0.264^{* *}$ & & 4.48 & 1.04 \\
\hline 6) Empathy & $0.328^{\star *}$ & $0.349^{* *}$ & $0.480^{* *}$ & $0.369^{* *}$ & $0.417^{* *}$ & 5.41 & 0.79 \\
\hline
\end{tabular}

${ }^{*} P<0.05,{ }^{*} P<0.01, S D$ Standard deviation, $P I$ Purchase intention, IND Independence self-construal, INT interdependence self-construal, $M I I$ Moral identity internalization, MIS Moral identity symbolization. 
and interference effect of moral identity were verified based on the bootstrap method proposed by Hayes (2009), which was deemed more persuasive than the Sobel test or causal steps approach proposed by Baron and Kenny (1986) (MacKinnon et al., 2004; Williams \& MacKinnon, 2008; Hayes, 2009). Repeated sampling was performed 5,000 times using the bootstrap method to obtain the upper and lower bounds of the trust interval. The indirect effect was established when 0 was not included in the trust interval (Hayes, 2009). Because independent and interdependent self-construal are two sets of self-schemata that exist simultaneously (Markus \& Kitayama, 1991), the present study investigated the separate impacts of these two types of self-construal on purchase intention toward CRM products.

\subsubsection{Impact of Independent Self-Construal on Purchase Intention toward CRM Products}

According to the regression analysis results, the direct effect of consumers' independent self-construal on their purchase intention toward CRM products was nonsignificant $(\mathrm{t}=1.7159, p=0.0877)$, and thus, H1a was not supported. Independent self-construal exerted a significant impact on empathy $(\beta=0.4591, p<$ $0.0001)$ and empathy exerted a significant impact on purchase intention $(\beta=$ 1.5705, $p=0.0002$ ). The coefficient of conditional indirect effects in Table 3 further confirmed that the mediating effect of empathy was significant, and thus H2a was supported.

The moderation effect of moral identity is illustrated in Table 3. The "low/ low" and "low/high" groups of moral identity internalization and symbolization exerted significant direct moderation effects on independent self-construal and the purchase intention (95\% confidence interval [CI], $0.1374-0.8881$ and 0.2083 1.2612), and thus $\mathrm{H} 3 \mathrm{a}$ and $\mathrm{H} 4 \mathrm{a}$ were supported. However, the moderation effects

Table 3. Direct and indirect moderation effects of moral identity on independent selfconstrual and the purchase intention.

\begin{tabular}{ccccccc}
\hline & & & & \multicolumn{2}{c}{ Product of coefficients } & \multicolumn{2}{c}{$\begin{array}{c}\text { Bootstrap 5000 times } \\
\text { Confidence Interval }\end{array}$} \\
\cline { 4 - 7 } MII & MIS & Measure paths & Estimate & SE & Lower & Upper \\
\hline L & L & IND $\rightarrow$ PI & 0.5127 & 0.1904 & 0.1374 & 0.8881 \\
L & H & IND $\rightarrow$ PI & 0.7348 & 0.2671 & 0.2083 & 1.2612 \\
H & L & IND $\rightarrow$ PI & 0.0191 & 0.2426 & -0.4591 & 0.4973 \\
H & H & IND $\rightarrow$ PI & 0.2411 & 0.1959 & -0.1450 & 0.6273 \\
L & L & IND $\rightarrow$ Empathy $\rightarrow$ PI & 0.2946 & 0.1028 & 0.1274 & 0.5245 \\
L & H & IND $\rightarrow$ Empathy $\rightarrow$ PI & 0.2262 & 0.1461 & -0.4603 & 0.2742 \\
H & L & IND $\rightarrow$ Empathy $\rightarrow$ PI & -0.0635 & 0.1843 & -0.0045 & 0.5886 \\
H & H & IND $\rightarrow$ Empathy $\rightarrow$ PI & -0.1318 & 0.1099 & -0.3966 & 0.0429 \\
\hline
\end{tabular}

MII Moral identity internalization, MIS Moral identity symbolization, PI Purchase intention, $L$ Low, $H$ High, IND Independence self-construal. 
of moral identity symbolization in the high score groups were nonsignificant ( $95 \% \mathrm{CI},-0.4591-0.4973$ and $-0.1450-0.6273)$. In addition, the indirect moderation effects of moral identity internalization and symbolization in the "low/low" groups on independent self-construal and purchase intention achieved significance (95\% CI,0.1274 - 0.5245), and thus H5a and H6a were supported, although the moderation effects of the other moral identity subgroups were nonsignificant.

\subsubsection{Impact of Interdependent Self-Construal on Purchase Intention toward CRM Products}

The regression analysis results indicated that the direct effect of interdependent self-construal on purchase intention toward CRM products was nonsignificant $(\mathrm{t}=$ 0.2493, $p=0.8034)$, and thus H1b was not supported. Interdependent self-construal exerted a significant impact on empathy $(\beta=0.7309, p<0.0001)$ and empathy exerted a significant impact on purchase intention $(\beta=1.7499, \mathrm{p}<0.0001)$. The coefficient of conditional indirect effects in Table 4 further confirmed that the mediating effect of empathy was significant, and thus $\mathrm{H} 2 \mathrm{~b}$ was supported.

Concerning the moderation effect of moral identity, Table 4 shows that apart from the direct moderation effect of the "high/low" groups from moral identity internalization and symbolization on interdependent self-construal and purchase intention, which was nonsignificant (95\% CI, $-1.1574-0.1395)$, the remaining groups all achieved significance, and thus $\mathrm{H} 3 \mathrm{~b}$ and $\mathrm{H} 4 \mathrm{~b}$ were supported. In addition, apart from the "low/high" groups from moral identity internalization and symbolization, which exerted a nonsignificant direct moderation effect on interdependent self-construal and purchase intention (95\% CI, $-0.9785-0.0673)$, the remaining groups all achieved significance. Notably, the negative effects of

Table 4. The direct and indirect moderation effect of moral identity on interdependent self-construal and the purchase intention.

\begin{tabular}{ccccccc}
\hline & & & & & & \\
Product of coefficients & \multicolumn{2}{c}{$\begin{array}{c}\text { Bootstrap 5000 times } \\
\text { Confidence Interval }\end{array}$} \\
\cline { 4 - 7 } & MIS & Measure paths & Estimate & SE & Lower & Upper \\
\hline L & L & INT $\rightarrow$ PI & 0.4513 & 0.2118 & 0.0338 & 0.8687 \\
L & H & INT $\rightarrow$ PI & 1.7379 & 0.3307 & 1.0860 & 2.3898 \\
H & L & INT $\rightarrow$ PI & -0.5089 & 0.3289 & -1.1574 & 0.1395 \\
H & H & INT $\rightarrow$ PI & 0.7777 & 0.2276 & 0.3290 & 1.2264 \\
L & L & INT $\rightarrow$ Empathy $\rightarrow$ PI & 0.4446 & 0.1555 & 0.1859 & 0.7798 \\
L & H & INT $\rightarrow$ Empathy $\rightarrow$ PI & -0.4414 & 0.2668 & -0.9785 & 0.0673 \\
H & L & INT $\rightarrow$ Empathy $\rightarrow$ PI & 0.4963 & 0.2198 & 0.1310 & 1.0337 \\
H & H & INT $\rightarrow$ Empathy $\rightarrow$ PI & -0.3897 & 0.1662 & -0.7771 & -0.1172 \\
\hline
\end{tabular}

MII Moral identity internalization, MIS Moral identity symbolization, PI Purchase intention, $L$ Low, $H$ High, INT interdependence self-construal. 
the "high/high" groups from moral identity internalization and symbolization on the indirect moderation effect of interdependent self-construal and purchase intention was observed ( $\beta=-0.3897,95 \% \mathrm{CI},-0.7771--0.1172$ ), whereas the moderation effects of the remaining groups were positive. Thus, H5b and H6b were not supported.

\subsubsection{Impact of Dominant Self-Construal on Purchase Intention toward CRM Products}

Because consumers' independent and interdependent self-construal were two sets of simultaneously existing self-schemata (Markus \& Kitayama, 1991), this study further referenced the long-term self-construal method in past studies, where the mean score of interdependent self-construal items is first deducted from that of independent self-construal items. A higher score represents that independent self-construal is the dominant self-construal, whereas a lower score represents that interdependent self-construal is the dominant self-construal (Kitayama et al., 2009). The regression analysis results of the present study demonstrated that the direct effect of the dominant self-construal on purchase intention toward CRM products was nonsignificant $(\mathrm{t}=1.6096, p=0.1090)$.

Regarding the moderation effect of moral identity, the direct moderation effect of each group from moral identity internalization and symbolization on the dominant self-construal and purchase intention was nonsignificant. In regard to the indirect moderation effect, apart from the "low/high" group from moral identity internalization and symbolization, which achieved a nonsignificant direct moderation effect on the dominant self-construal and purchase intention (95\% CI, $-0.9785-0.0673)$, the remaining groups all achieved significance. Notably, the "low/low" and "high/low" groups from moral identity internalization and symbolization, which exerted a negative (weakened) effect on the direct moderation effect of the dominant self-construal and purchase intention on the dominant self-construal and purchase intention $(\beta=-0.1578,95 \% \mathrm{CI},-0.3509$ -0.0333 and $\beta=-0.1545,95 \% \mathrm{CI},-0.457--0.0196$, respectively), exerted a positive (strengthened) effect though the moderation effect of the "high/high" group $(\beta=0.0911,95 \%$ CI, $0.0107-0.2876)$.

\section{Conclusions and Recommendations}

The empirical results revealed the following findings. First, in the case of the "dual dimensions" of independent and interdependent self-construal, the direct impact of moral identity internalization and symbolization on interdependent self-construal toward purchase intention toward CRM products produced a positive moderation effect. However, under the indirect influence of empathy, the "low/low" and "high/high" groups from moral identity internalization and symbolization yielded positive and negative moderation effects, respectively. Second, regarding the direct impact of moral identity internalization and symbolization on independent self-construal toward purchase intention toward CRM products, the "low/low" and "low/high" groups from the two dimensions 
produced positive moderation effects. However, under the indirect influence of empathy, only the "low/low" group produced a significant positive moderation effect. Interdependent self-construal and independent self-construal produced different outcomes regarding purchase intention toward CRM products under the indirect influence of empathy. One reason for this could be that interdependent self-construal emphasizes the self in social contexts (Duclos \& Barasch, 2014). High moral identity internalization and symbolization may have a higher demand on moral actions and cast doubt on the causes linked to CRM (Sasse \& Trahan, 2007; Varadarajan \& Menon, 1988), thereby producing a weakened interference effect on the impact of empathy, a topic that future studies are recommended to explore.

In addition, when this study further converted self-construal into a single dominant dimension, independent self-construal was discovered to be the dominant self-construal and did not have a significant direct impact on purchase intention toward CRM products. However, under the mediating effect of empathy, the "low/low" and "high/high" groups from moral identity internalization and symbolization each produced negative and positive moderation effects. The finding was notable because according to Winterich, Mittal, and Aquino (2013), recognition did not exhibit an incentive effect on charitable actions. However, the results in the present study indicated that the "low/low" group from moral identity internalization and symbolization produced a weakening moderation effect on the mediating effect of empathy toward self-construal and purchase intention toward CRM products. However, whether this result is unique to Chinese society requires verification from subsequent in-depth investigation and empirical research.

In terms of theoretical contributions, with the consumers' self-construal and its impact on purchase intention toward CRM products, this study constructed the mediating role of empathy and moderated mediation role of the two dimensions of moral identity in this influential relationship. Because few studies have identified the significance of symbolization (He et al., 2016; Lee et al., 2014; Xie et al., 2015), the present study simultaneously explored the joint impacts of the double dimensions of moral identity internalization and symbolization and of empathy on purchase intention toward CRM products, which was an extended contribution of moral identity theory. In addition, owing to the divergence in the results of the impact of self-construal on consumer decision-making (Hong \& Lee, 2010; Hong \& Chang, 2015), the present study conducted an in-depth analysis of the impact of cognitive and emotional factors on consumption decisions, thereby responding to scholars' calls for studies on the impact of emotional factors on consumer behavior (Griskevicius et al., 2010). Furthermore, the present study proposed that self-construal is the antecedent variable that affects moral emotions. Empirical studies have revealed that under the influence of the collectivist culture in Taiwan, the two dimensions of moral identity and the single dominant dimension exerted distinct moderation effects on the mediating 
effect of empathy, thereby constituting crucial theoretical contributions related to cognitive and emotional variables.

In regard to practical recommendations, this study discovered that under the indirect (mediation) effect of empathy on interdependent self-construal, the "low/low" and "high/high" groups from moral identity internalization and symbolization each produced positive and negative moderation effects. Therefore, companies are recommended to perform market segmentation based on consumers' moral identities, where emotional marketing strategies can be used for the "low/low" groups of internalization and symbolization to evoke consumer empathy. Because the "high/high" group produces a weakening effect on the mediating effect of empathy, this group was speculated to possibly have doubts and distrust toward the motivations and causes of corporate CRM, thereby resulting in a perception of cause-exploitative marketing (Sasse \& Trahan, 2007; Varadarajan \& Menon, 1988). Thus, companies must employ more sincere marketing strategies for "high/high" groups to reduce consumer doubt toward CRM motives. Different marketing strategies for different market segments are strongly recommended.

Concerning research limitations, this study merely explored the reaction of long-term self-construal to CRM. Future studies are recommended to conduct experimental designs to investigate whether situational self-construal has different reactions to CRM. In addition, because the two dimensions of moral identity-internalization and symbolization-were positively correlated, future studies are recommended to verify whether they interact with each other (Aquino \& Reed, 2002), as well as their potential moderation effects on the emotional variable.

\section{References}

Aaker, J., \& Williams, P. (1998). Empathy versus Pride: The Influence of Emotional Appeals across Cultures. Journal of Consumer Research, 25, 241-261. https://doi.org/10.1086/209537

Andrew, M., Luo, X., Fang, Z., \& Aspara, J. (2014). Cause Marketing Effectiveness and the Moderating Role of Price Discounts. Journal of Marketing, 78, 120-142. https://doi.org/10.1509/jm.14.0003

Aquino, K., \& Reed, A. I. I. (2002). The Self-Importance of Moral Identity. Journal of Personality and Social Psychology, 83, 1423-1440. https://doi.org/10.1037/0022-3514.83.6.1423

Aquino, K., Freeman, D., Reed, A. I. I., Lim, V. K. G., \& Felps, W. (2009). Testing a Social Cognitive Model of Moral Behaviour: The Interactive Influence of Situation and Moral Identity Centrality. Journal of Personality and Social Psychology, 97, 123-141. https://doi.org/10.1037/a0015406

Arnett, D. B., German, S. D., \& Hunt, S. D. (2003). The Identity Salience Model of Relationship: The Case of Nonprofit Marketing. Journal of Marketing, 13, 785-802.

Bandura, A. (1989). Social Cognitive Theory of Organizational Management. Academy of Management Review, 14, 361-384.

Baron, R. M., \& Kenny, D. A. (1986). The Moderator-Mediator Variable Distinction in 
Social Psychological Research: Conceptual, Strategic, and Statistical Considerations. Journal of Personality and Social Psychology, 51, 1173-1182. https://doi.org/10.1037/0022-3514.51.6.1173

Barone, M. J., \& Roy, T. (2010). The Effect of Deal Exclusivity on Consumer Response to Targeted Price Promotions: A Social Identification Perspective. Journal of Consumer Psychology, 20, 78-89. https://doi.org/10.1016/j.jcps.2009.10.002

Batson, C. D. (1987). Prosocial Motivation: Is It Ever Truly Altruistic? Advances in Experimental Social Psychology, 20, 65-122. https://doi.org/10.1016/S0065-2601(08)60412-8

Bhattacharya, C., Korschun, D., \& Sen, S. (2009). Strengthen Stakeholder-Company Relationships through Mutually Beneficial Corporate Social Responsibility Initiatives. Journal of Business Ethics, 85, 257-272. https://doi.org/10.1007/s10551-008-9730-3

Commonwealth (2016). http://www.cw.com.tw/article/article.action?id=5077792

Duclos, R., \& Barasch, A. (2014). Prosocial Behavior in Intergroup Relations: How Donor Self-Construal and Recipient Group-Membership Shape Generosity. Journal of Consumer Research, 41, 93-108. https://doi.org/10.1086/674976

Eisenberg, N., \& Miller, P. A. (1987). The Relation of Empathy to Prosocial and Related Behaviors. Psychological Bulletin, 101, 91-119. https://doi.org/10.1037/0033-2909.101.1.91

Eisenberg, N., Valiente, C., \& Champion, C. (2004). Empathy-Related Responding: Moral, Social, and Socialization Correlates. In A. G. Miller (Eds.), The Social Psychology of Good and Evil (pp. 386-415). New York, NY: Guilford.

Forehand, M. R., \& Grier, S. (2003). When Is Honesty the Best Policy? The Effect of Stated Company Intent on Consumer Skepticism. Journal of Consumer Psychology, 13, 349-356. https://doi.org/10.1207/S15327663JCP1303_15

Gelman, A., \& Park, D. K. (2009). Splitting a Predictor at the Upper Quarter or Third and the Lower Quarter or Third. The American Statistician, 63, 1-8. https://doi.org/10.1198/tast.2009.0001

Godfrey, P. C. (2005). The Relationship between Corporate Philanthropy and Shareholder Wealth: A Risk Management Perspective. The Academy of Management Review, 30, 777-798. https://doi.org/10.5465/AMR.2005.18378878

Griskevicius, V., Shiota, M. N., \& Nowlis, S. M. (2010). The Many Shades of Rose-Colored Glasses: An Enolutionary Approach to the Influence of Different Positive Emotions. Journal of Consumer Research, 37, 238-250. https://doi.org/10.1086/651442

Haidt, J. (2003). The Moral Emotions. In R. J. Davidson, K. R. Scherer, \& H. H. Goldsmith (Eds.), Handbook of Affective Sciences (pp. 852-870). Oxford: Oxford University Press.

Hayes, A. F. (2009). Beyond Baron and Kenny: Statistical Mediation Analysis in the New Millennium. Communication Monographs, 76, 408-420. https://doi.org/10.1080/03637750903310360

Hayes, A. F. (2013). An Introduction to Mediation, Moderation, and Conditional Process Analysis: A Regression-Based Approach. New York, NY: Guilford Press.

He, H., Zhu, W., Gouran, D., \& Kolo, O. (2016). Moral Identity Centrality and Cause-Related Marketing: The Moderating Effects of Brand Social Responsibility Image and Emotional Brand Attachment. European Journal of Marketing, 50, 236-259. https://doi.org/10.1108/EJM-10-2014-0613

Ho, D. Y. F. (1995). Selfhood and Identity in Confucianism, Taoism, Buddhism, and Hinduism: Constructs with the West. Journal for the Theory of Social Behavior, 25, 115-139. https://doi.org/10.1111/j.1468-5914.1995.tb00269.x 
Hoffman, M. L. (1981). The Development of Empathy. In J. P. Rushton, \& R. M. Sorrentino (Eds.), Altruism and Helping Behavior: Social, Personality, and Developmental Perspectives (pp. 41-63). Erlbaum, NJ: Hillsdale.

Hoffman, M. L. (2000). Empathy and Moral Development. Implications for Caring and Justice. Cambridge: Cambridge University Press. https://doi.org/10.1017/CBO9780511805851

Hofstede, G. (2001). Culture's Consequences (2nd ed.). Thousand Oaks, CA: Sage.

Hong, J., \& Chang, H. H. (2015). "I” Follow My Heart and "We” Rely on Reasons: The Impact of Self-Construal on Reliance on Feelings versus Reasons in Decision Making. Journal of Consumer Research, 41, 1392-1411. https://doi.org/10.1086/680082

Hong, J., \& Lee, A. Y. (2010). Feeling Mixed but Not Torn: The Moderating Role of Construal Level in Mixed Emotions Appeals. Journal of Consumer Research, 37, 456-472. https://doi.org/10.1086/653492

Huang, K. K. (1987). Face and Favour: The Chinese Power Game. American Journal of Sociological Society, 92, 944-974.

Kelly, T. L. (1939). The Selection of Upper and Lower Groups for the Validation of Test Items. Journal of Educational Psychology, 30, 17-24. https://doi.org/10.1037/h0057123

Kim, J.-E., \& Johnson, K. K. P. (2013). The Impact of Moral Emotions on Cause-Related Marketing Campaigns: A Cross-Cultural Examination. Journal of Business Ethics, 112, 79-90. https://doi.org/10.1007/s10551-012-1233-6

Kitayama, S., Markus, H. R., \& Kurokawa, M. (2000). Culture, Emotion, and Well-Being: Good Feelings in Japan and the United States. Cognition and Emotion, 14, 93-124. https://doi.org/10.1080/026999300379003

Kitayama, S., Mesquita, B., \& Karasawa, M. (2006). Cultural Affordances and Emotional Experience: Socially Engaging and Disengaging Emotions in Japan and the United States. Journal of Personality and Social Psychology, 91, 890-903.

https://doi.org/10.1037/0022-3514.91.5.890

Kitayama, S., Park, H., Sevincer, A. T., Karasawa, M., \& Uskul, A. K. (2009). A Cultural Task Analysis of Implicit Independence: Comparing North America, Western Europe, and East Asia. Journal of Personality and Social Psychology, 97, 236-255. https://doi.org/10.1037/a0015999

Krishna, A., \& Rajan, U. (2009). Cause Marketing: Spillover Effects of Cause-Related Products in a Product Portfolio. Management Science, 55, 1469-1485. https://doi.org/10.1287/mnsc.1090.1043

Lafferty, B. A., \& Goldsmith, R. E. (2005). Cause-Brand Alliances: Does the Cause Help the Brand or Does the Brand Help the Cause? Psychology \& Marketing, 58, 423-429. https://doi.org/10.1016/j.jbusres.2003.07.001

Lazarus, R. S. (1991). Emotion and Adaptation. New York, NY: Oxford University Press.

Lee, H., Park, T., Moon, H. K., Yang, Y., \& Kim, C. (2009). Corporate Philanthropy, Attitude towards Corporations, and Purchase Intentions: A South Korea Study. Journal of Business Research, 62, 939-946. https://doi.org/10.1016/j.jbusres.2008.08.007

Lee, S., Winterich, K. P., \& Ross Jr., W. T. (2014). I'm Moral, But I Won't Help You: The Distinct Roles of Empathy and Justice in Donations. Journal of Consumer Research, 41, 678-696. https://doi.org/10.1086/677226

Leszczyc, P. T. L. P., \& Rothkopf, M. H. (2010). Charitable Motives and Bidding in Charity Auctions. Management Science, 56, 399-413.

https://doi.org/10.1287/mnsc.1090.1120

Lu, L. (2003). Defining the Self-Other Relation: The Emergence of a Composite Self. In- 
digenous Psychological Research in Chinese Societies, 20, 139-207.

Lu, L., Chang T. T., \& Chang, Y. Y. (2012). The Meaning of Work and Family and Its Role in Coping with Work and Family Conflict: Practicing the Chinese Bicultural Self. Indigenous Psychological Research in Chinese Societies, 37, 141-189.

MacKinnon, D. P., Lockwood, C. M., \& Williams, J. (2004). Confidence Limits for the Indirect Effect: Distribution of the Product and Re-Sampling Methods. Multivariate Behavioral Research, 39, 99-128. https://doi.org/10.1207/s15327906mbr3901_4

Markus, H., \& Kitayama, S. (1991). Culture and the Self: Implications for Cognition, Emotion and Motivation. Psychological Review, 98, 224-253. https://doi.org/10.1037/0033-295X.98.2.224

Matsumoto, D. (1989). Cultural Influences on the Perception of Emotion. Journal of Cross-Cultural Psychology, 20, 92-105. https://doi.org/10.1177/0022022189201006

Oyserman, D. (2009). Identity-Based Motivation: Implications for Action-Readiness, Procedural-Readiness, and Consumer Behavior. Journal of Consumer Psychology, 19, 250-260. https://doi.org/10.1016/j.jcps.2009.05.008

Reed, A. (2004). Activating the Self-Importance of Consumer Selves: Exploring Identity Salience Effect on Judgments. Journal of Consumer Research, 31, 286-295. https://doi.org/10.1086/422108

Reed, A., \& Aquino, K. (2003). Moral Identity and the Expanding Circle of Moral Regard toward Out-Groups. Journal of Personality and Social Psychology, 84, 1270-1286. https://doi.org/10.1037/0022-3514.84.6.1270

Reed, A., Aquino, K., \& Levy, E. (2007). Moral Identity and Judgments of Charitable Behaviours. Journal of Marketing, 71, 178-193. https://doi.org/10.1509/jmkg.71.1.178

Ross, J. K., Patterson, L. T., \& Stutt, M. A. (1992). Consumers Perceptions of Organizations That Use Cause-Related Marketing. Journal of Academy of Marketing Science, 20, 93-97. https://doi.org/10.1007/BF02723480

Sasse, C. M., \& Trahan, R. T. (2007). Rethinking the New Corporate Philanthropy. Business Horizon, 50, 29-38. https://doi.org/10.1016/j.bushor.2006.05.002

Shen, H., Wan, F., \& Wyer, R. S. (2011). Cross-Cultural Differences in the Refusal to Accept a Small Gift: The Differential Influence of Reciprocity Norms on Asians and North Americans. Journal of Personality and Social Psychology, 100, 271-281. https://doi.org/10.1037/a0021201

Singelis, T. M. (1994). The Measurement of Independent and Interdependent Self-Con struals. Personality and Social Psychology Bulletin, 20, 580-591. https://doi.org/10.1177/0146167294205014

Skarmeas, D., \& Shabbir, H. A. (2011). Relationship Quality and Giving Behaviour in the UK Fundraising Sector: Exploring the Antecedent Roles of Religiosity and Self-Construal. European Journal of Marketing, 45, 720-738. https://doi.org/10.1108/03090561111120000

Tangney, J. P., Stuewig, J., \& Mashek, D. J. (2007). Moral Emotions and Moral Behavior. The Annual Review of Psychology, 58, 345-372. https://doi.org/10.1146/annurev.psych.56.091103.070145

Thomas, M. L., Mullen, L. G., \& Fraedrich, J. (2011). Increased Word-of-Mouth via Strategic Cause-Related Marketing. International Journal of Nonprofit and Voluntary Sector Marketing, 16, 36-49. https://doi.org/10.1002/nvsm.393

Vaidyanathan, R., Aggarwal, P., \& Kozlowski, W. (2013). Interdependent Self-Construal in Collectivist Cultures: Effects on Compliance in a Cause-Related Marketing Context. Journal of Marketing Communications, 19, 44-57. 
https://doi.org/10.1080/13527266.2011.551833

Varadarajan, P. R., \& Menon, A. (1988). Cause-Related Marketing: A Coalignment of Marketing Strategy and Corporate Philanthropy. Journal of Marketing, 52, 58-74. https://doi.org/10.2307/1251450

White, K., MacDonnell, R., \& Ellard, J. H. (2012). Belief in a Just World: Consumer Intentions and Behaviors toward Ethical Products. Journal of Marketing, 76, 103-118. https://doi.org/10.1509/jm.09.0581

Williams, J., \& MacKinnon, D. P. (2008). Resampling and Distribution of the Product Methods for Testing Indirect Effects in Complex Models. Structural Equation Modelling, 15, 23-51. https://doi.org/10.1080/10705510701758166

Winterich, K. P., \& Barone, M. (2011). Warm Glow or Cold, Hard Cash? Social Identity Effects on Consumer Choice for Donation versus Discount Promotions. Journal of Marketing Research, 48, 855-868. https://doi.org/10.1509/jmkr.48.5.855

Winterich, K. P., Aquino, K., Mittal, V., \& Swartz, R. (2013). When Moral Identity Symbolization Motives Prosocial Behavior: The Role of Recognition and Moral Identity Internalization. Journal of Applied Psychology, 98, 759-770.

https://doi.org/10.1037/a0033177

Winterich, K. P., Mittal, V., \& Aquino, K. (2013). When Does Recognition Increase Charitable Behavior? Toward a Moral Identity-Based Model. Journal of Marketing, 77, 121134. https://doi.org/10.1509/jm.11.0477

Xie, C., Bagozzi, R. P., \& Gronhaug, K. (2015). The Role of Moral Emotions and Individual Differences in Consumer Responses to Corporate Green and Non-Green Action. Journal of the Academy of Marketing Science, 43, 333-356. https://doi.org/10.1007/s11747-014-0394-5

Yen, G. F., Wang, R. Y., \& Yang, H. T. (2017). How Consumer Mindsets in Ethnic Chinese Societies affect the Intention to Buy Fair Trade Products: The Mediating and Moderating Roles of Moral Identity. Asia Pacific Journal of Marketing and Logistics, 29, 1-18. https://doi.org/10.1108/APJML-06-2016-0107 